\title{
Induced Magnetic Ordering by Proton Irradiation in Graphite
}

\author{
P. Esquinazi * D. Spemann, R. Höhne, A. Setzer, K.-H. Han, and T. Butz \\ Institut für Experimentelle Physik II, Universität Leipzig, Linnéstr. 5, D-04103 Leipzig, Germany
}

\begin{abstract}
We provide evidence that proton irradiation of energy $2.25 \mathrm{MeV}$ on highly-oriented pyrolytic graphite samples triggers ferro- or ferrimagnetism. Measurements performed with a superconducting quantum interferometer device (SQUID) and magnetic force microscopy (MFM) reveal that the magnetic ordering is stable at room temperature.
\end{abstract}

The search for macroscopic magnetic ordering phenomena in organic materials has been pushed to the frontiers of physics, chemistry and materials science since the discovery of ferromagnetism in the open-shell radical $p$-nitrophenylnitronyl nitroxide ( $p-\mathrm{NPNN})$ [1] and in tetrakis(dimethylamino)ethylene $[\mathrm{TDAE}]{ }^{+} \mathrm{C}_{60}$ chargetransfer salt [2] with Curie temperatures $T_{c}=0.6 \mathrm{~K}$ and $16 \mathrm{~K}$, respectively. Although a remarkable amount of studies have been performed in the last years building molecules with unpaired $\pi$-electrons [3], the scientific community did not become aware of the possible existence of a room temperature metal-free organic ferromagnet till the accidental "discovery" of ferromagnetism in polymerized fullerenes was reported [4, 5]. The study of the magnetic properties of polymerized fullerene was triggered by the previously reported weak ferromagnetic-like magnetization loops in highly-oriented pyrolytic graphite (HOPG) 6] that indicate the existence of magnetic ordering with $T_{c}$ much above room temperature [7]. The origin for the reported ferro- or ferrimagnetism in polymerized $\mathrm{C}_{60}$ as well as in HOPG remains unclear. The contribution of ferromagnetic impurities, like iron, has been thoroughly investigated [7, [8] and it is something one should carefully take into account, specially when the ferromagnetic signal under study is small. Ferromagnetism in a graphite nodule from a meteorite with $T_{c} \simeq 570 \mathrm{~K}$ has been recently suggested to be due to a large magnetic proximity effect at the interface with magnetite or kamacite inclusions [9]. As in Ref. 9] most of the magnetism specialists doubt that a room-temperature magnet can exist in a material with only s- and p-electrons. Clear evidence for its existence and reproducibility as well as the report of a relatively easy method to produce it, is of fundamental interest for a broad spectrum of natural sciences.

Early reports on room-temperature ferromagnetic behavior in some carbon-based structures were apparently not taken seriously by the scientific community, partially due to the weakness of the ferromagnetic signals added to the unknown contribution of magnetic impurities and low reproducibility [10]. From those early works our attention was focused to the magnetic properties found in amorphous-like carbon prepared from different hydrogenrich starting materials where an increase of the saturation magnetization with the hydrogen concentration in the starting material was found [1]. The origin for the mag- netic ordering has been related to the mixture of carbon atoms with $\mathrm{sp}^{2}$ and $\mathrm{sp}^{3}$ bonds, which was predicted to reach a magnetization higher than in pure Fe 12. Hydrogen, on the other hand, was assumed to have a role only in the formation of the amorphous carbon structure 11]. New theoretical predictions, however, show that hydrogenated graphite can display spontaneous magnetization coming from different numbers of mono- and dihydrogenated carbon atoms [13]. We show in this letter that implanted protons in HOPG triggers ferromagnetic (or ferrimagnetic) ordering with a Curie temperature above room temperature.

Two HOPG (ZYA grade) samples from Advanced Ceramics Co. with a content of magnetic metallic impurities below $1 \mathrm{ppm}$ [7] were irradiated with a $2.25 \mathrm{MeV}$ proton microbeam. The samples' dimension was $2 \times 2 \times 0.1 \mathrm{~mm}^{3}$. Before irradiation they were glued with varnish on a high-purity Si substrate and the magnetic moment of the whole ensemble as well as of the Si substrate alone were measured. Due to the small sample mass the contribution of the HOPG virgin sample ferromagnetic signal (saturation magnetization $M_{s} \sim 3 \times 10^{-4} \mathrm{emu} / \mathrm{g}$ at temperatures $T \leq 300 \mathrm{~K}[7]$ ) is overwhelmed by the diamagnetic signal of the Si-substrate for fields applied parallel to the graphene layers. Rutherford Backscattering Spectroscopy and Particle Induced X-ray Emission spectra were recorded simultaneously with the irradiation allowing us to check the purity of the sample at the different irradiation stages. Assuming that all the (measured) Fe impurities $(<0.3 \mu \mathrm{g}$ Fe per gram graphite) in our samples behave as bulk ferromagnetic material, a rather unrealistic assumption, their maximum contribution to the magnetic moment in our samples would be $m<6.1 \times 10^{-8} \mathrm{emu}$. The irradiated and non-irradiated areas were further characterized by atomic force and magnetic force microscopy (AFM,MFM) with a Nanoscope III scanning probe microscope operating in "tapping/lift" mode, using standard tips coated with a magnetic $\mathrm{CoCr}$ film magnetized normal to the sample surface. The magnetization measurements were performed with a SQUID magnetometer from Quantum Design with the reciprocating sample option (RSO) and a sensitivity of $\lesssim 10^{-7} \mathrm{emu}$.

Four irradiations were consecutively applied to sample 1 , namely: stage $\# 1$ : homogeneous irradiation of an area $1720 \times 1720 \mu \mathrm{m}^{2}$, dose: $0.99 \mathrm{pC} / \mu \mathrm{m}^{2}$, total charge: 2.93 
$\mu \mathrm{C} ; \# 2: 100 \times 100$ spots of $2 \mu \mathrm{m}$ diameter each, on an area $570 \times 570 \mu \mathrm{m}^{2}$ in the middle of the sample, dose: 0.3 $\mathrm{nC} / \mu \mathrm{m}^{2}$, total charge: $\simeq 8 \mu \mathrm{C} ; \# 3: 4$ spots of $0.8 \mathrm{~mm}$ diameter each, dose: $0.3 \mathrm{nC} / \mu \mathrm{m}^{2}$, total charge: $\simeq 600 \mu \mathrm{C}$; \#4: the same as \#3. To check the reproducibility of our procedure as well as to rule out possible contamination during the handling of the sample, a new piece of the virgin $\mathrm{HOPG}$ sample was prepared in a similar way and fixed to a different Si-substrate. On this sample 2 three spots of $0.8 \mathrm{~mm}$ diameter, dose: $0.3 \mathrm{nC} / \mu \mathrm{m}^{2}$, total charge: $450 \mu \mathrm{C}$ were irradiated, similar to stage \#3 but one spot less.

To estimate the defect density created by the proton beam, Monte Carlo simulations (SRIM2003 [14]) were performed with full damage cascades assuming a displacement energy of $E_{d}=35 \mathrm{eV}$ for the creation of a Frenkel pair in graphite. According to these calculations, $2.25 \mathrm{MeV}$ protons come to rest at a depth of $\sim 46 \mu \mathrm{m}$ from the graphite surface with a $1.75 \mu \mathrm{m}$ width (full width at half maximum of the implantation profile). Since hydrogen is fully trapped in HOPG up to a concentration of $0.45 \mathrm{H} / \mathrm{C}$ [15], all implanted protons will be trapped for doses up to $14 \mathrm{nC} / \mu \mathrm{m}^{2}$ when they come to rest in the HOPG sample. Assuming that no annealing of defects during irradiation occurs and that the damaged regions are of the size of the irradiated one, we obtain that for proton dose of $0.3 \mathrm{nC} / \mu \mathrm{m}^{2}$ the defect density is $\sim 2 \times 10^{20} \mathrm{~cm}^{3}$, i.e. only $\sim 0.1 \%$ of the carbon atoms are displaced. For a dose 250 times higher we expect that $35 \%$ of the carbon atoms are displaced and the carbon lattice is amorphous.

Figure 1(a) shows the measured magnetic moment of sample 1 after irradiation \#1, which is similar to the virgin sample within the scale of the figure. The field was applied parallel to the graphene planes. The main part ( $\sim 90 \%)$ of the diamagnetic signal is due to the Si substrate. In the same figure we show the magnetic moment of the same ensemble after irradiation \#3 where we can clearly recognize the s-shape curve, see Fig. 1(a), without any background substraction. After subtraction of the magnetic moment of the substrate we obtain for sample 1 the results depicted in Fig. 1(b). We observe a clear increase of the ferromagnetic loop with irradiation steps $\# 2$ and \#3. For the last step \#4 the magnetic moment of the irradiated sample decreased slightly. The coercive fields are between 80 and 110 Oe and weak temperature dependent. The remanent magnetic moments are approximately $20 \%$ of the values of the saturation magnetic moments. For the sample with the highest magnetic moment (sample 1 after stage \#3): $m_{\text {rem }} \simeq 1 \times 10^{-6}$ emu.

The observed changes of the magnetic moment after the different irradiation stages are due to the irradiation and not to different misalignments of the sample position respect to the applied field. To check this we measured sample 1 in the irradiation stage \#3 and \#4 for the other field direction (parallel to the c-axis of graphite). After
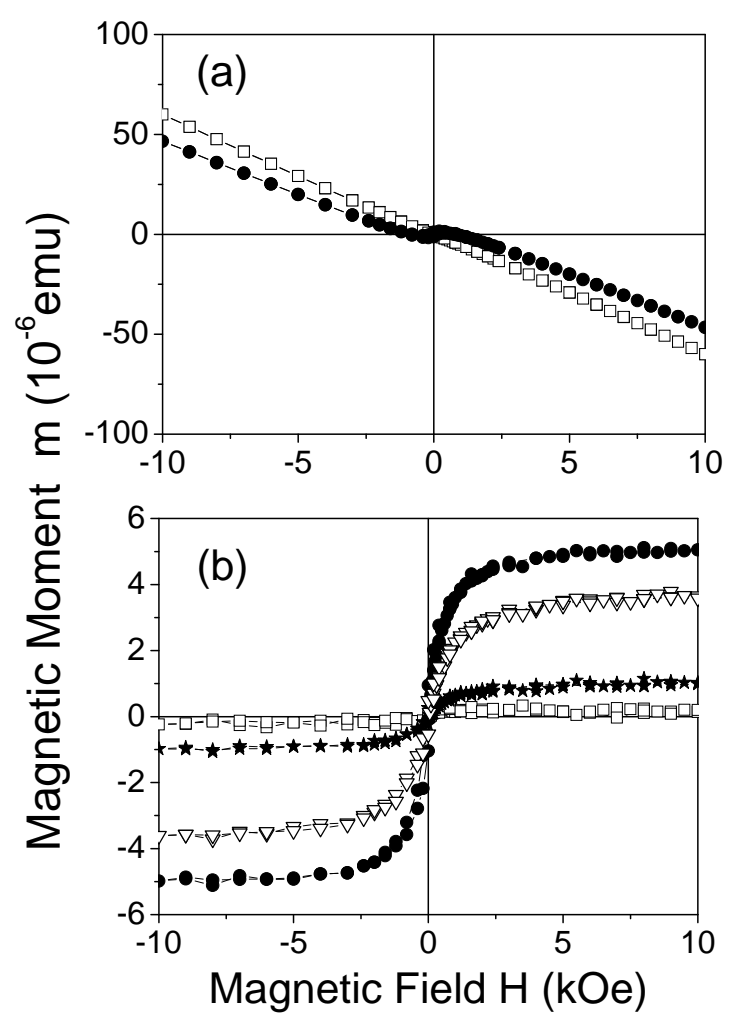

FIG. 1: The magnetic moment (in units of $10^{-6} \mathrm{emu}=$ $10^{-9} \mathrm{Am}^{2}$ ) measured at $T=300 \mathrm{~K}$ as a function of magnetic field $\left(1 \mathrm{kOe}=10^{6} / 4 \pi \mathrm{Am}^{-1}\right)$ by cycling the field from zero to $+10 \mathrm{kOe}$, from $+10 \mathrm{kOe}$ to $-10 \mathrm{kOe}$ and back to $+10 \mathrm{kOe}$ for sample 1 glued on a silicon substrate, after various proton irradiations. (a) Total magnetic moment without any background subtraction for the sample after stage \#1 ( $\square$ ) and \#3 (•) irradiations. (b) Magnetic moment after subtraction of the sample holder magnetic moment, for the sample after the first $(\square)$, second $(\star)$, third $(\bullet)$ and fourth $(\nabla)$ irradiation stages.

subtraction of the diamagnetic signal from graphite and Si substrate, the magnetic moment loops were similar as for the other field direction; this is an indication for a low anisotropy of the magnetic ordering produced by the irradiation.

Figure 2(a) shows the magnetic moment of sample 2 before and after irradiation of the three spots. The background signal due to the substrate was subtracted. We see a clear development of the ferromagnetic signal after irradiation. The saturation magnetic moment is slightly smaller than the one we would expect assuming a linear relation between saturation moment $m_{s}$ and total implanted charge $C_{t}$, namely $\sim(3 / 4) \times 5 \times 10^{-6}=$ $3.8 \times 10^{-6} \mathrm{emu}$. This value is $10 \%$ larger than the measured value $m_{s} \simeq 3.5 \times 10^{-6} \mathrm{emu}$. Although the number of points is too small to provide with certainty the 

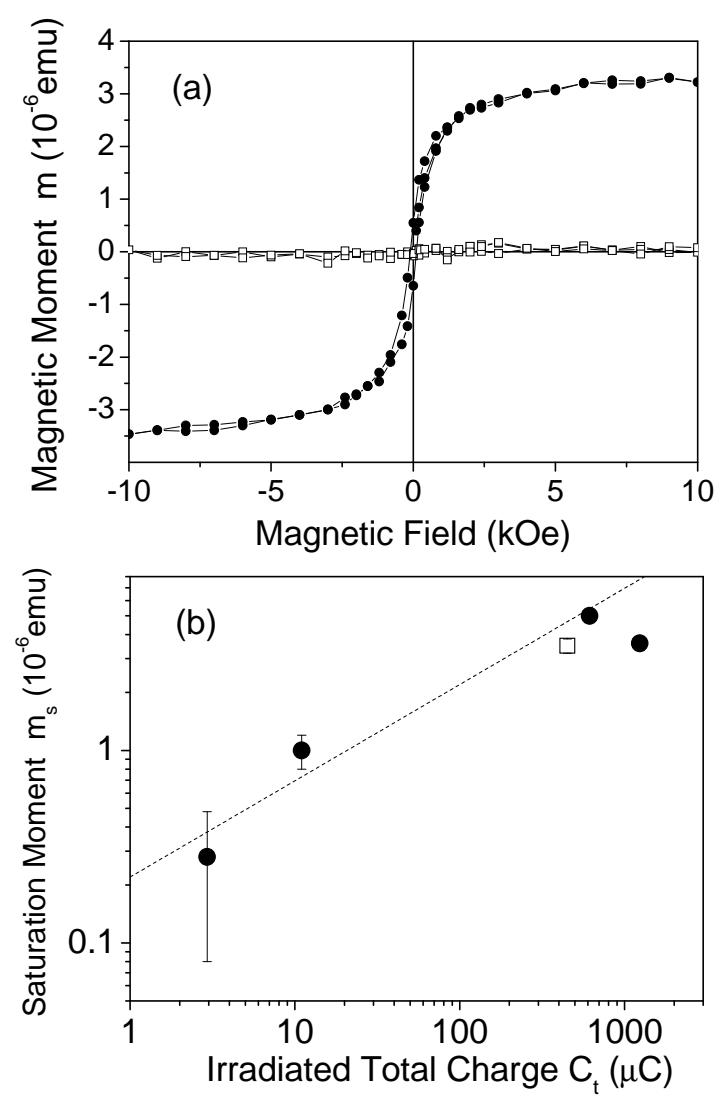

FIG. 2: (a) Magnetic moment (in units of $10^{-6} \mathrm{emu}$ ) measured at $T=300 \mathrm{~K}$ as a function of magnetic field as in Fig.1(b), for sample 2 before $(\square)$ and after (•) proton irradiation. (b) Measured saturation magnetic moment as a function of the total irradiated charge $C_{t}$ for sample 1 $(\bullet)$ and for sample $2(\square)$. The dashed line is the funtion $m_{s}=0.22\left[10^{-6} \mathrm{emu} / \mu \mathrm{C}^{0.5}\right] C_{t}^{0.5}$.

relationship between $m_{s}$ and $C_{t}$, our results indicate a relation of the type $m_{s} \propto \sqrt{C_{t}}$, see Fig. 2(b). It is interesting to note that after the \#4 irradiation the magnetic moment of sample 1 decreases, see Fig. 1(a), indicating that there might be a competition between the produced disorder and the implanted charge, which determines the total magnetic ordering.

Hysteresis loops were measured at $5 \mathrm{~K}, 300 \mathrm{~K}$ and $380 \mathrm{~K}$. In this temperature range there is no significant change of the ferromagnetic loops with temperature. This result is similar to that obtained for the "intrinsic" ferromagnetic-like loops of graphite in Ref. 7], and indicates a Curie temperature much above $400 \mathrm{~K}$, which is of importance for future applications.

The MFM pictures obtained at the irradiated areas show homogeneously distributed magnetic domains, as depicted in Fig. 3. The periodicity and/or the width of the domains depends on the irradiation dose, ranging from $4 \mu \mathrm{m}$ in the second irradiated stage area to

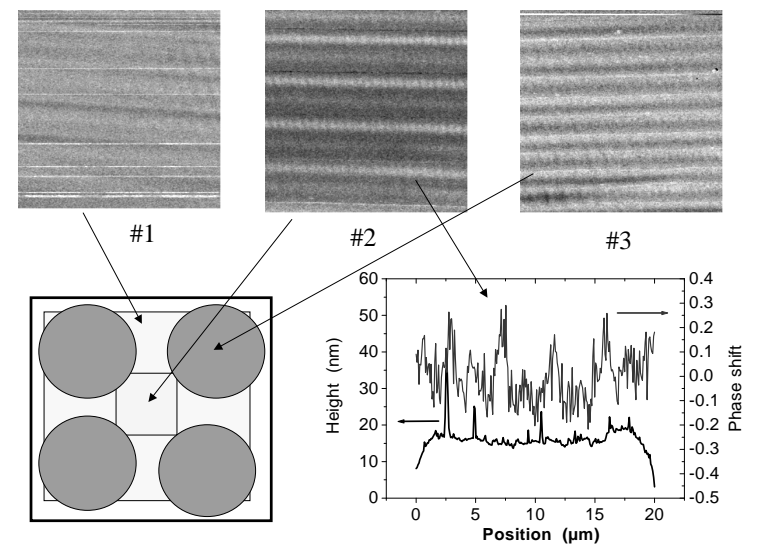

FIG. 3: Top: Phase gradient pictures obtained from MFM at three surfaces of sample 1 corresponding to the irradiation stages \#1, \#2 and \#3, see sketch at the bottom left of the figure. Bottom right: Topography and phase gradient line scans of the middle MFM picture obtained at stage \#2.

about $2 \mu \mathrm{m}$ at the area of the third stage. A weak domain structure is recognized at the area of stage \#1, see Fig. 3 We stress that the magnetic signal is not correlated to the topography in any irradiation stage. For comparison, in Fig. [3 we show a topography and magnetic line-scans obtained normal to the magnetic domain structure at stage \#2. The MFM pictures shown in Fig. 3 were obtained at a distance of $50 \mathrm{~nm}$ between tip and surface. Due to the small coercive field of the magnetic surface and the influence of the magnetic tip we have observed that the magnetic domain distribution depends on the distance between tip and surface. Theoretically, the phase shift $\Delta \phi$ measured by MFM should be proportional to the magnetic force gradient, which should depend on the distance between the tip apex and sample surface $h$ as $(h+\delta)^{-5}$, being $\delta$ the distance between tip apex and the effective position of the magnetic moment in the tip. Measuring $\Delta \phi$ as a function of $h$ we estimate $\delta \sim(900 \pm 100) \mathrm{nm}$. With this value we estimate the maximum magnetic moment observed by the tip $m \sim 3 \times 10^{-15} \mathrm{Am}^{2}$ at the area of largest magnetic moment (stages \#3 and \#4). We note that measurements on different magnetic samples indicate that $\delta$ depends on the magnetic properties of the measured surface and therefore the calculated magnetic moment from MFM data should be taken as a rough estimate only [16]. Assuming that the magnetic moment measured by MFM corresponds to a region of area $\sim 10^{-12} \mathrm{~m}^{2}$ and $\sim 1 \mu \mathrm{m}$ depth, we can estimate the total saturation magnetic moment $m_{s}$ the SQUID would measure at stage \#3 as $m_{s} \sim\left[3 \times 10^{-15} \times 4 \times \pi \times\left(0.4 \times 10^{-3}\right)^{2} / 10^{-12}\right] 10^{3} \mathrm{emu}=$ $6 \times 10^{-6} \mathrm{emu}$, in reasonable agreement with the SQUID measurements.

Although a proton penetration depth of $\sim 46 \mu \mathrm{m}$ is 
expected, we do not know how large is the depth for the ferromagnetic active part of the irradiated sample. This will be studied by irradiating samples of different thickness in the future. If we assume a depth of $1 \mu \mathrm{m}$ we get a magnetization $M \simeq 1.1 \mathrm{emu} / \mathrm{g}$ for sample 1 after stage $\# 3$, which is $\sim 1 \%$ of that of magnetite and of the same order as that obtained for amorphous-like carbon prepared by CVD from hydrogen-rich targets 11]. From MFM, the maximum phase shift observed in the irradiated regions is of the order of $\Delta \phi_{\max } \sim 0.3^{\circ}$. From measurements on regions with Fe impurities (mainly $\mathrm{Fe}_{3} \mathrm{O}_{4}$ of micrometer size) with the same tip and arrangement [16] we obtain $\Delta \phi_{\max } \sim 10^{\circ} \ldots 20^{\circ}$. That means that $\Delta \phi_{\max }$ obtained in the irradiated regions $\# 3$ is of the order of $1 \%$ of that of magnetite, in agreement with the rough estimate made above using the SQUID data and assuming $1 \mu \mathrm{m}$ ferromagnetic active thickness.

Magnetic force microscopy measurements on protonirradiated spots on HOPG show that it is possible to create ferromagnetic active areas of $\sim 1 \mu \mathrm{m}^{2}$ with very sharp borders of the magnetic force gradient, created mainly at low proton doses [17]. That means that it is possible to produce magnetic microstructures of arbitrary shape by a dedicated proton beam scanning system. To check the importance of hydrogen, we have produced spots of similar topography with $1.5 \mathrm{MeV}$ helium ion irradiation at different doses $\left(>0.05 \mathrm{nC} / \mu \mathrm{m}^{2}\right)$. Those irradiated regions showed no significant magnetic signal in MFM and stress the significance of hydrogen in the formation of the magnetic ordering in graphite 17].

From SQUID and MFM measurements we conclude that magnetic ordering appears after proton irradiation in graphite and that this is stable at room temperature. Our results provide a possible answer to the origin of ferromagnetism in other carbon-based structures [4, 8, 9], which is not correlated with magnetic impurities. Future experiments should clarify the lattice position of hydrogen in graphite as well as its role in the magnetic ordering. The characterization of our samples with MicroRaman as well as XPS is of particular interest and will be performed in the future. Systematic investigations of the magnetic properties of different carbon materials irradiated at different ion doses are necessary to gain more insight into the physical processes involved as well as to broader the spectrum for future applications. MFM measurements on proton irradiated amorphous-carbon films indicate the existence of weak magnetic ordering. As possible explanation for our results we refer to the work in Ref. [13] where a sp ${ }^{3}-\mathrm{sp}^{2}$ ferrimagnetic structure can arise in a graphene plane through the existence of monoand dihydrogenated carbon atoms. Future work should provide an answer to the question to what extent the graphite structure represents a quantitative advantage for the formation of the magnetic ordering.

Our experimental findings open up a new research field in magnetism with possible applications in spin electronics, since according to Ref. [13] the electrons in the hydrogenated graphene should have a very large spin polarization. If the interpretation of hydrogen-induced ferrimagnetism in HOPG 13] is correct, then with our technique it should be possible to create the smallest controlled magnet ever possible by implanting hydrogen at the edges of carbon nanotubes.

One of the authors (P.E.) thanks K. Kusakabe for correspondence. This work is supported by DFG Grant ES 86/6-3.

* E-mail address: esquin@physik.uni-leipzig.de

[1] P. Turek et al., Chem. Phys. Lett. 180, 327 (1991); M. Tamura et al., Chem. Phys. Lett. 186, 401 (1991)

[2] P. M. Allemand et al., Science 253, 301 (1991).

[3] For recent reviews see: $\pi$-Electron Magnetism: From Molecules to Magnetic Materials, J. Veciana (ed.), Structure and Bonding Vol. 100, Springer Verlag Berlin (2001).

[4] T. Makarova et al.,Nature 413, 716 (2001).

[5] R. A. Wood et al., J. Phys.: Condens. Matter 14, L385 (2002).

[6] Y. Kopelevich, P. Esquinazi, J.H.S. Torres, and S. Moehlecke, J. Low Temp. Phys. 119, 691 (2000).

[7] P. Esquinazi et al., Phys. Rev. B 66, 024429 (2002).

[8] R. Höhne and P. Esquinazi, Adv. Mater. 14, 753 (2002).

[9] J. Coey et al., Nature 420, 156 (2002).

[10] T.Makarova in Studies of High-Temperature Superconductivity, A. Narlikar (ed.), NOVA Science Publishers, Inc. (New York), Vol. 45 (2003), p. 107.

[11] K. Murata, H. Ueda, and K. Kawaguchi, Synth. Met. 44, 357 (1991). K. Murata, H. Ushijima, H. Ueda, K. Kawaguchi, J. Chem. Soc., Chem. Commun. 18, 1265 (1991); ibid 7, 567 (1992).

[12] A. A. Ovchinnikov and V. N. Spector, Synth. Metals 27, B615 (1988).

[13] K. Kusakabe and M. Maruyama, Phys. Rev. B 67, 092406 (2003).

[14] J. F. Ziegler (ed.), The stopping and range of ions in matter, Vol.2-6, Pergamon Press, New York (1977-1985).

[15] R. Siegele, J. Roth, B. M. U. Scherzer, and S. J. Pennycook, J. Appl. Phys. 73, 2225 (1993).

[16] K.-H. Han, D. Spemann, R. Höhne, A. Setzer, T. Makarova, P. Esquinazi, and T. Butz, Carbon 41, 785(2003) and Addendum, Carbon 41, 2427 (2003).

[17] K.-H. Han, D. Spemann, P. Esquinazi, R. Höhne, V. Riede and T. Butz, Advanced Materials (2003, in press). 